\title{
EUROfusion
}

WPMST1-PR(17) 18397

M Faitsch et al.

\section{Dependence of the L-Mode scrape-off layer power fall-off length on the upper triangularity in TCV}

Preprint of Paper to be submitted for publication in Plasma Physics and Controlled Fusion

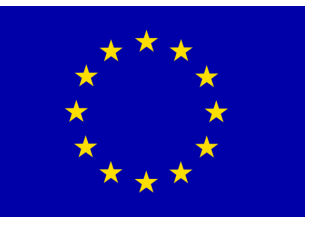

This work has been carried out within the framework of the EUROfusion Consortium and has received funding from the Euratom research and training programme 2014-2018 under grant agreement No 633053. The views and opinions expressed herein do not necessarily reflect those of the European Commission. 
This document is intended for publication in the open literature. It is made available on the clear understanding that it may not be further circulated and extracts or references may not be published prior to publication of the original when applicable, or without the consent of the Publications Officer, EUROfusion Programme Management Unit, Culham Science Centre, Abingdon, Oxon, OX14 3DB, UK or e-mail Publications.Officer@euro-fusion.org

Enquiries about Copyright and reproduction should be addressed to the Publications Officer, EUROfusion Programme Management Unit, Culham Science Centre, Abingdon, Oxon, OX14 3DB, UK or e-mail Publications.Officer@euro-fusion.org

The contents of this preprint and all other EUROfusion Preprints, Reports and Conference Papers are available to view online free at http://www.euro-fusionscipub.org. This site has full search facilities and e-mail alert options. In the JET specific papers the diagrams contained within the PDFs on this site are hyperlinked 


\title{
Dependence of the L-Mode scrape-off layer power fall-off length on the upper triangularity in TCV
}

\author{
M.Faitsch $^{1}$, R.Maurizio ${ }^{2}$, A.Gallo ${ }^{3}$, S.Coda ${ }^{2}$, T.Eich ${ }^{1}$, \\ B.Labit ${ }^{2}$, A.Merle ${ }^{2}$, H.Reimerdes ${ }^{2}$, B.Sieglin ${ }^{1}$, C.Theiler ${ }^{2}$, \\ the Eurofusion MST1 Team ${ }^{4}$ and the TCV Team ${ }^{5}$ \\ ${ }^{1}$ Max-Planck-Institute for Plasma Physics, Boltzmannstr. 2, D-85748 Garching, \\ Germany \\ 2 Ecole Polytechnique Federale de Lausanne, Swiss Plasma Center, CH-1015 \\ Lausanne, Switzerland \\ 3 CEA Cadarache, IRFM, F-13108 Saint-Paul-Lez-Durance, France \\ 4 See author list of "H. Meyer et al 2017 Nucl. Fusion 57 102014" \\ ${ }^{5}$ See author list of "S. Coda et al 2017 Nucl. Fusion 57 102011" \\ E-mail: Michael.Faitsch@ipp.mpg.de
}

\begin{abstract}
This paper reports on experimental observations on TCV with a scan in upper triangularity $\delta_{\text {up }}$, including negative triangularity, focusing on the power fall-off length $\lambda_{\mathrm{q}}$ in L-Mode. The upper triangularity is scanned from -0.28 to 0.47 . Smaller $\lambda_{\mathrm{q}}^{\text {out }}$ is measured at the outer divertor target for decreasing $\delta_{\mathrm{up}}$ together with higher edge temperature $T_{\mathrm{e}}$,edge leading to increased confinement. This effect is observed for both magnetic drift directions for discharges in deuterium and helium. In helium larger $\lambda_{\mathrm{q}}$ values are observed compared to deuterium. The power fall-off length at the inner divertor target $\lambda_{\mathrm{q}}^{\text {in }}$ has a non monotonic behaviour with changing triangularity. The largest values are around $\delta_{\text {up }}=0$. The ratio $\lambda_{\mathrm{q}}^{\text {in }} / \lambda_{\mathrm{q}}^{\text {out }}$ increases for decreasing $\delta_{\mathrm{up}}$ for positive triangularity and is approximately constant for negative triangularity. $\lambda_{\mathrm{q}}^{\text {out }}$ is compared to available scaling laws. Partial agreement is only observed for a scaling law containing a proxy for $T_{\text {e,edge }}$ at ASDEX Upgrade [Sieglin, PPCF 2016]. Extending this scaling to TCV and using $T_{\mathrm{e}, \text { edge }}$ at $\rho_{\mathrm{pol}}=0.95$ suggests that $\lambda_{\mathrm{q}}^{\text {out }}$ is independent of machine size $\lambda_{\mathrm{q}}^{\mathrm{L}-\text { Mode }}[\mathrm{mm}]=165 \cdot B_{\mathrm{pol}}[\mathrm{T}]^{-0.66} \cdot A[\mathrm{u}]^{-0.15} \cdot T_{\mathrm{e}, \text { edge }}[\mathrm{eV}]^{-0.93}$. $R[\mathrm{~m}]^{-0.03}$. Possible explanations for smaller $\lambda_{\mathrm{q}}^{\text {out }}$ for decreasing $\delta_{\mathrm{up}}$ is a reduction in turbulence or a direct effect of increasing $T_{\mathrm{e}, \text { edge }}$.
\end{abstract}

\section{Introduction}

Understanding and controlling power exhaust is an active area of research in the tokamak community. Divertor heat loads in next step fusion devices like ITER are expected to be close to or exceeding material limits $[1,2]$ making significant impurity seeding necessary, the amount depends on the scrape-off layer width $[3,4]$. Though scaling laws for inter-ELM transport are well accepted, an agreed physics picture of near scrape-off layer transport is under discussion. This paper reports on experimental studies aiming at (i) understanding the effect of plasma shaping and reversing toroidal magnetic field polarity to support further theoretical work, e.g. the importance of drifts and turbulence on scrape-off layer heat flux and (ii) scaling the effect of negative triangularity for accessing the reactor design with negative triangularity $[5,6,7]$. The Tokamak à Configuration Variable (TCV) [8, 9] is able to achieve a large variety 
of plasma shapes due to 16 independently powered poloidal field coils and an open vessel geometry. TCV is commonly used to investigate shaping effects, e.g. plasma triangularity $\delta$, and alternative divertor configurations in view of DEMO. The machine is equipped with carbon plasma facing components. Recent power exhaust studies in TCV feature a variety of shaping and higher order null configurations (e.g. snowflake) are discussed in $[10,11,12]$. The effect of divertor leg length on the heat flux profile is discussed in $[13,14,15]$.

This paper focuses on the effect of plasma triangularity on heat flux on both divertor targets for a single null configuration with particular emphasis on negative triangularity. A triangularity dependence of the power fall-off length $\lambda_{\mathrm{q}}$ in combination with reversed toroidal field has been observed in ASDEX Upgrade [16]. Negative triangularity has been investigated in $\operatorname{TCV}[17,18]$ and reactor studies $[5,6,7]$. Such investigations showed increased energy confinement with negative triangularity in low [19] and medium [20] density plasmas, explained by a reduction of turbulent transport [21].

A second aspect of the presented study is the influence of the ion $\nabla$ B drift direction on scrape-off layer transport. Heat flux studies with reversed field, i.e. reversed $\nabla \mathrm{B}$ drift direction, were performed in, e.g., ASDEX Upgrade [22, 23, 16], EAST [24, 25] and JET [26] and demonstrated the importance of vertical drifts in determining the heat flux profiles.

The third objective is to study the influence of the main ion species on the power fall-off length $\lambda_{\mathrm{q}}$. This is experimentally tackled by comparing deuterium and helium plasmas. A larger $\lambda_{\mathrm{q}}$ is reported for ASDEX Upgrade [27] and JET [28, 29] operating in helium compared to deuterium.

The paper is organized as follows: the database and global discharge parameters including experimental correlations between upper triangularity $\delta_{\text {up }}$ and selected edge parameters are presented in section 2. In section 3 experimental heat flux analysis from IR are shown for the outer and inner target. The experimental results are discussed in section 3 with a comparison to available scaling laws, predictions from the heuristic drift-based model by Goldston [30], turbulent transport [31] as well as implications for a negative triangularity reactor design. Summary and conclusions are given in section 5 .

\section{Upstream and target conditions as function of upper triangularity}

The database used for this study consists of ohmically heated L-Mode discharges, in deuterium (D) and helium (He) in standard lower single null divertor configuration. For each main ion species, discharges with both toroidal field directions were performed. A negative (positive) toroidal field corresponds to the downwards (upwards) ion drift direction and will be referred to as favourable (non-favourable) drift direction towards (away from) the X-point, fav (non). The direction of plasma current is changed together with the magnetic field direction to keep plasma helicity. The range of global plasma parameters is shown in table 1. Absolute values of plasma current and toroidal magnetic field are $240 \mathrm{kA}$ and $1.43 \mathrm{~T}$, respectively. Plasma current and toroidal magnetic field are $240 \mathrm{kA}$ and $1.43 \mathrm{~T}$, respectively. The direction of plasma current is changed together with the magnetic field direction to keep plasma helicity. Plasma shape for both species is altered by scanning upper triangularity $\delta_{\text {up }}$ between -0.28 and +0.47 ( +0.60 for helium and non-favourable drift direction). The plasma separatrix given by the magnetic equilibrium reconstruction code LIUQE [32] 
together with profiles of parallel connection length in the scrape-off layer to each target for four discharges are shown in figure 1 . The lower part of the magnetic configuration is not changed, keeping the divertor geometry fixed. While the connection length between outer mid-plane and outer target is not changing during the $\delta_{\text {up }}$ scan, the connection length to the inner target varies mainly because of the decreasing distance, mapped to the outer mid-plane, between the active (lower) and non-active (upper) X-points. The vertical lines in figure 1(b) and figure 1(c) mark the position $\left(R-R_{\mathrm{sep}}=5 \mathrm{~mm}\right)$ at which the parallel connection length $L_{\mathrm{OMP}}$ is taken in further discussions. $L_{\mathrm{OMP}}$ is taken $5 \mathrm{~mm}$ away from the separatrix in the scrape-off layer where the length does not change significantly anymore with increasing distance [14], except for discharges with the secondary X-point at a poloidal flux surface close to the separatrix. This choice is further motivated by the smallest power fall-off length $\lambda_{\mathrm{q}}^{\text {out }} \approx 5 \mathrm{~mm}$ measured at the outer divertor target within this database. The outer mid-plane is defined as the outermost point of the separatrix w.r.t. major radius $R$. The elongation $\kappa$ at the normalized poloidal flux surface $\rho_{\text {pol }}=0.95$ is around 1.4 with
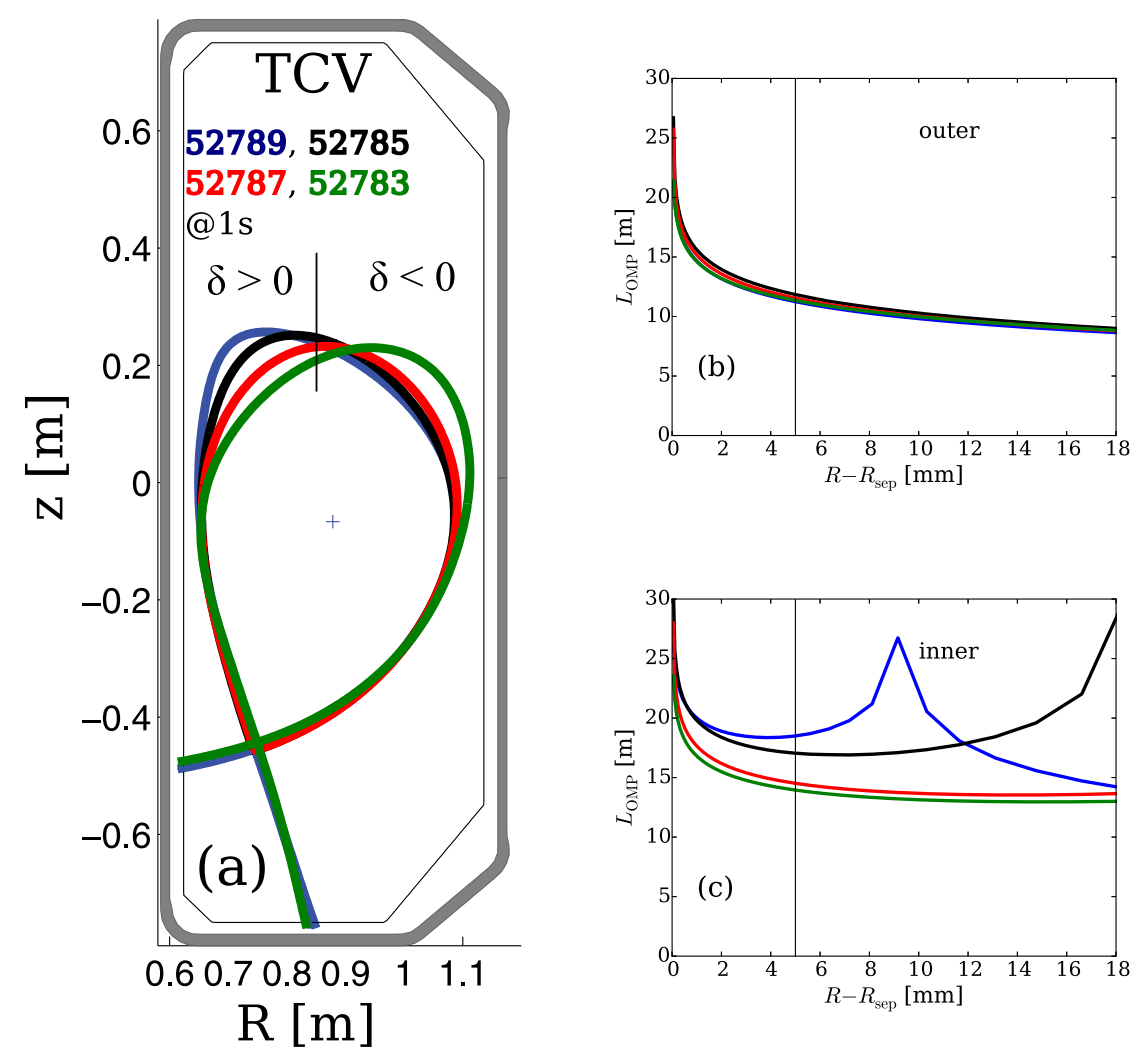

Figure 1. (a) Poloidal cross section and radial profiles of connection length to (b) outer and (c) inner target at the outer mid-plane for four different upper triangularities.

a variation between 1.28 and 1.53 with smaller average $\kappa$ for $\delta_{\text {up }}<0$ (figure 2(a)). The database for deuterium contains additional discharges with lower $\kappa$. The edge safety 
factor $q_{95}$ varies between 2.5 and 3.5, figure 2(b). Plasma density is kept constant
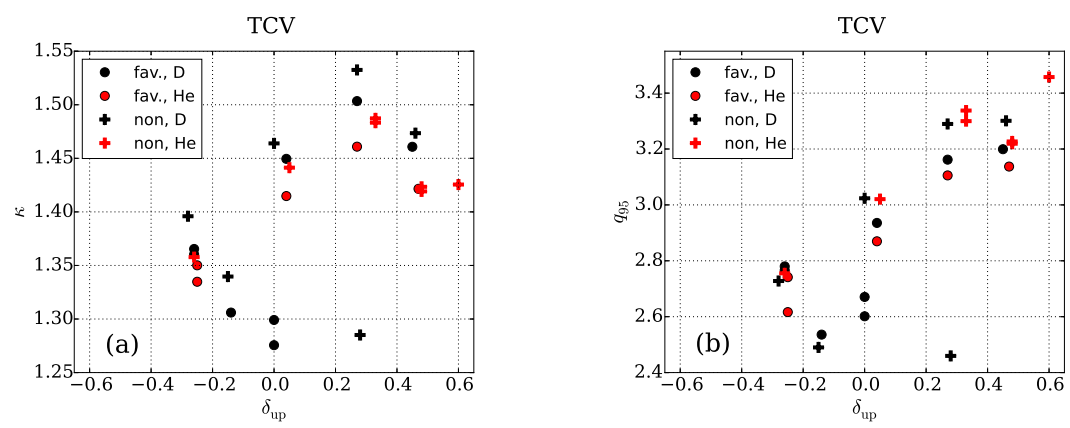

Figure 2. Correlation of upper triangularity $\delta_{\text {up }}$ with (a) elongation $\kappa$ and (b) edge safety factor $q_{95}$.

during discharges but varies between discharges. The density is kept low enough to be fully attached (peak target electron temperature above $10 \mathrm{eV}$ ) but high enough to avoid strong MHD activity. The line averaged electron density $n_{\mathrm{e}, \text { avg }}$ ranges between $4-8 \cdot 10^{19} \mathrm{~m}^{-3}$ with on average larger values for helium plasmas compared to deuterium (figure 3(c)).

The power crossing the separatrix $P_{\mathrm{SOL}}$ is estimated by subtracting the radiated power in the confined region $P_{\text {rad,main }}$ deduced from tomographic inversion of bolometry [33] from the ohmic power $P_{\mathrm{ohm}}$ from the equilibrium reconstruction code LIUQE [32]. $P_{\mathrm{SOL}}$ is in the range of $200 \pm 50 \mathrm{~kW}$ for all discharges in this database without correlation to $\delta_{\text {up }}$.

Edge electron temperature $T_{\text {e,edge }}$ is measured using a Thomson scattering system

Table 1. Discharge Parameters of the database.

\begin{tabular}{|c|c|c|c|c|c|c|c|c|}
\hline Ion & Drift & $\begin{array}{c}\mathbf{B}_{\text {tor }} \\
{[\mathrm{T}]}\end{array}$ & $\begin{array}{c}\mathbf{I}_{\mathrm{p}} \\
{[\mathrm{kA}]}\end{array}$ & $\begin{array}{c}\mathbf{n}_{\mathrm{e}, \mathrm{avg}} \\
{\left[10^{19} \mathrm{~m}^{-3}\right]}\end{array}$ & $\begin{array}{l}P_{\mathrm{SOL}} \\
{[\mathrm{kW}]}\end{array}$ & $\mathbf{q}_{95}$ & $\kappa$ & $\delta_{\text {up }}$ \\
\hline$\overline{\mathrm{D}}$ & & -1.43 & -240 & 4.1 to 6.2 & 170 to 230 & 2.5 to 3.2 & 1.3 to 1.5 & -0.26 to +0.45 \\
\hline $\mathrm{D}$ & nor & 1.43 & 240 & 4.4 to 7.0 & 160 to 200 & 2.5 to 3.3 & 1.3 to 1.5 & -0.28 to +0.46 \\
\hline $\mathrm{He}$ & fav. & -1.43 & -240 & 4.5 to 7.7 & 200 to 230 & 2.6 to 3.1 & 1.3 to 1.5 & -0.25 to +0.47 \\
\hline $\mathrm{He}$ & non & 1.43 & 240 & 5.4 to 6.5 & 180 to 200 & 2.8 to 3.5 & 1.3 to 1.5 & -0.26 to +0.60 \\
\hline
\end{tabular}

(TS) [34]. The value at $\rho_{\mathrm{pol}}=0.95$ is chosen as representation of the edge electron temperature of an L-Mode plasma, deduced from a linear fit to the plasma edge profiles within a time period of $200 \mathrm{~ms}$ with constant plasma parameters [35].

A clear correlation between $T_{\text {e,edge }}$ and $\delta_{\text {up }}$ is shown in figure 3 , which is independent of main ion species. However, the non-favourable drift direction exhibits higher $T_{\text {e,edge }}$ at similar $\delta_{\text {up }}$. Since, as mentioned before, $P_{\mathrm{SOL}}$ does not change in the database, the dependence of $T_{\mathrm{e}, \text { edge }}$ on $\delta_{\mathrm{up}}$ may indicate that the energy transport of the plasma decreases with decreasing (or negative) triangularity, consistent with previous studies $[20,19,21]$. The stored energy $W_{\mathrm{MHD}}$ increases for decreasing triangularity, too. The variation at fixed $\delta_{\text {up }}$ is mainly due to the plasma density, varying about a factor of two between individual discharges (figure 3(c)). The variation in $\delta_{\text {up }}$ changes $q_{95}$ and correspondingly connection length in the scrape-off layer. Connection length 

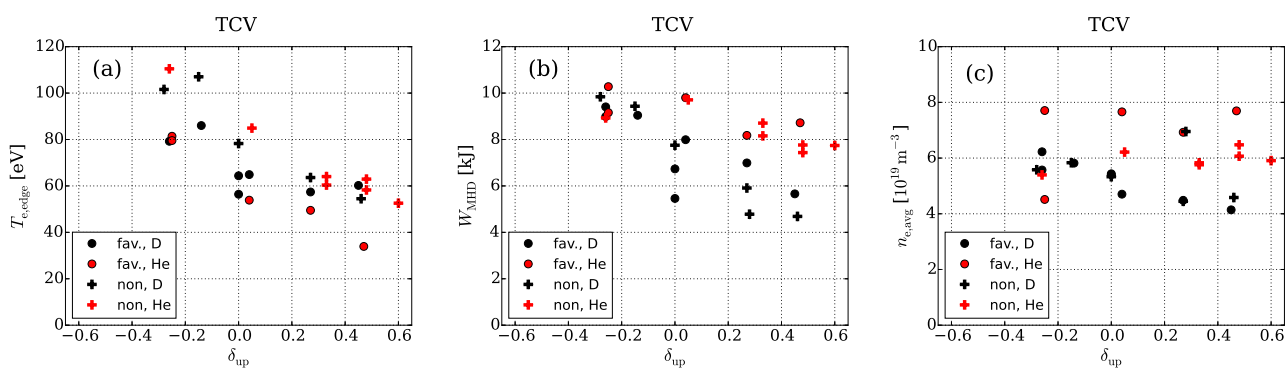

Figure 3. Correlation between upper triangularity $\delta_{\text {up }}$ and (a) edge electron temperature $T_{\text {e,edge }}$, (b) stored energy $W_{\mathrm{MHD}}$ and (c) line averaged density $n_{\mathrm{e}, \text { avg }}$.

$L_{\text {OMP }}^{\text {in }}$ to the inner target (figure $4(\mathrm{a})$ ) exhibits a pronounced change of more than $60 \%$ with changing $\delta_{\text {up }}$ as already noted in the discussion of figure $1(\mathrm{c})$. The effect is small
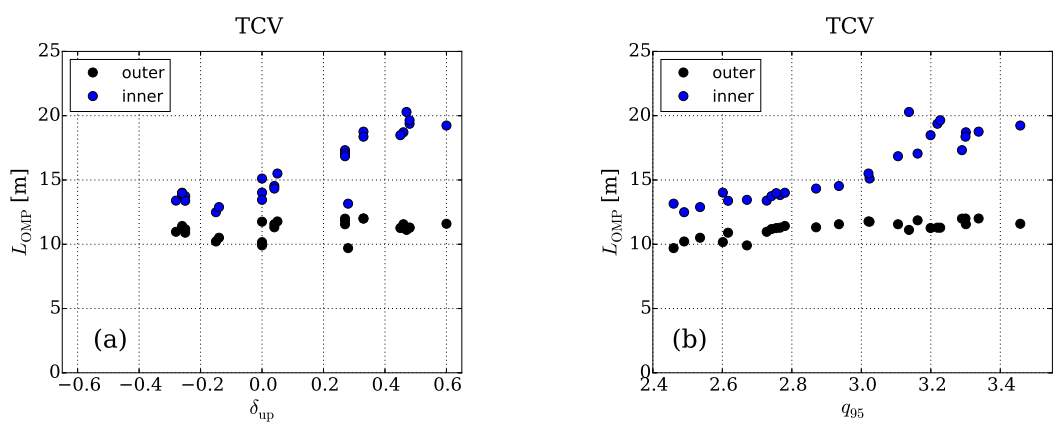

Figure 4. Connection length $L_{\mathrm{OMP}}$ from $5 \mathrm{~mm}$ outside the outermost point of the separatrix to both divertor targets versus (a) upper triangularity $\delta_{\text {up }}$ and (b) edge safety factor $q_{95}$.

(less than $25 \%$ ) for outer connection length $L_{\mathrm{OMP}}^{\text {out }}$. This change is correlated with the change in $q_{95}$, shown with comparing both $L_{\mathrm{OMP}}^{\text {in }}$ and $L_{\mathrm{OMP}}^{\text {out }}$ to $q_{95}$ in figure 4 (b).

The temperature evolution of both strike zones is measured using two infrared (IR) thermography systems [14]. Heat flux is calculated using the THEODOR code [36, 37]. Target electron temperature $T_{\mathrm{e}, \mathrm{t}}$ and density $n_{\mathrm{e}, \mathrm{t}}$ are estimated using Langmuir probes (LP) [38].

A power balance between $P_{\mathrm{ohm}}$ and sum of total radiated power from bolometry, $P_{\text {rad,tot }}$, and power to inner and outer divertor target plate, $P_{\text {in }}$ and $P_{\text {out }}$, calculated from profiles measured with the IR system, is shown in figure $5(\mathrm{c})$ depending on $\delta_{\text {up }}$. The power balance is in the range of 70-90\% except for the highest positive $\delta_{\text {up }}$ cases for which, being very close to double null, part of the power is deposited at nonmonitored areas.

The power on outer and inner target is shown in figure 5(a),(b) with no clear correlation with $\delta_{\text {up. }}$. It is seen that more power flows to the outer target, which is in line with previous studies[22, 26, 14].

In figure 6 peak target electron temperature for both outer, $T_{\mathrm{e}, \mathrm{t}}^{\mathrm{out}}$, and inner, $T_{\mathrm{e}, \mathrm{t}}^{\mathrm{in}}$, target are shown. Only heat flux profiles for peak temperatures above $10 \mathrm{eV}$ are used 

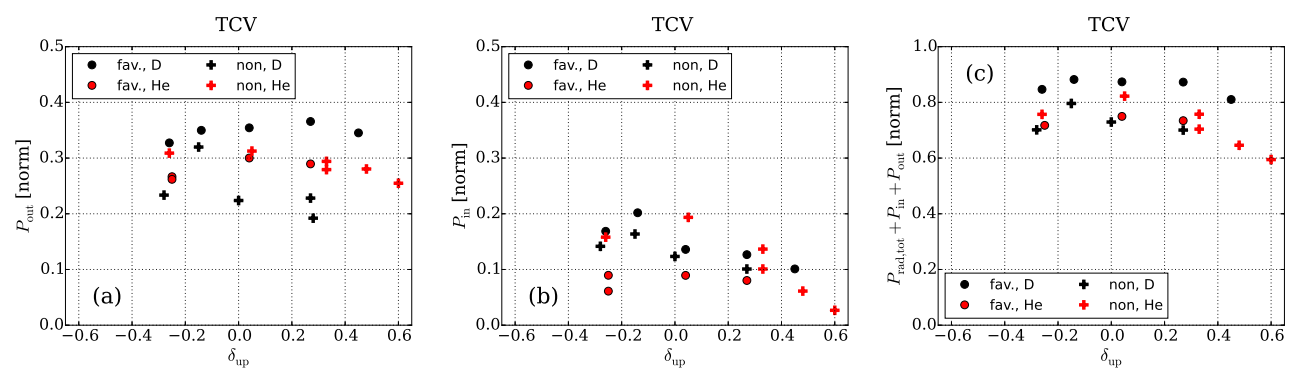

Figure 5. Power to the divertor target versus upper triangularity $\delta_{\text {up }}$ for (a) outer and (b) inner target and (c) power balance.
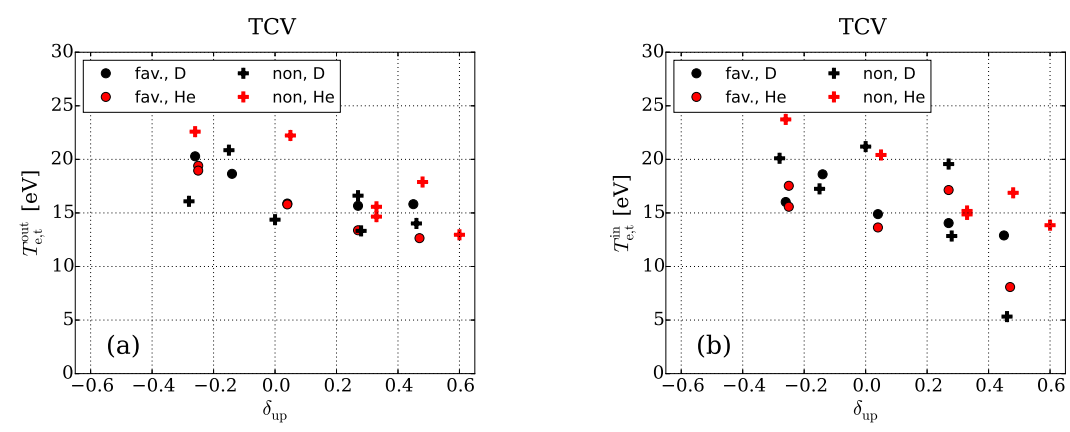

Figure 6. Correlation between target electron temperature $T_{\mathrm{e}, \mathrm{t}}$ and upper triangularity $\delta_{\text {up }}$ for (a) outer and (b) inner target.

for the database, assuring attached conditions. For this reason data for the inner target are excluded from two discharges since there $T_{\mathrm{e}, \mathrm{t}}^{\mathrm{in}}<10 \mathrm{eV}$.

\section{Divertor heat flux characterization}

The heat flux profile at the divertor target is described by the $1 \mathrm{D}$ diffusive model presented in [29]:

$$
q(s)=\frac{q_{0}}{2} \exp \left(\left(\frac{S}{2 \lambda_{\mathrm{q}}}\right)^{2}-\frac{s}{\lambda_{\mathrm{q}} f_{\mathrm{x}}^{*}}\right) \cdot \operatorname{erfc}\left(\frac{S}{2 \lambda_{\mathrm{q}}}-\frac{s}{S f_{\mathrm{x}}^{*}}\right)\left[\frac{\mathrm{W}}{\mathrm{m}^{2}}\right]
$$

with $s$ target location, $S$ divertor broadening, $\lambda_{\mathrm{q}}$ power fall-off length, $f_{\mathrm{x}}^{*}$ poloidal (effective) flux expansion between outer mid-plane and target and $q_{0}$ unbroadened peak heat flux density at the strike line position $(s=0)$.

Typical heat flux profiles for inner and outer divertor target are shown in figure 7 . The solid line represents a fit using (1) and corresponding values for $\lambda_{\mathrm{q}}$ and $S$ are presented. The flux expansion is calculated from equilibrium reconstruction.

The values for $\lambda_{\mathrm{q}}$ in the following are calculated using the method described in ref. [14]: The heat flux profile is mapped onto flux labels, converted into real space coordinates of the outer mid-plane. They are fitted afterwards, directly obtaining (upstream) $\lambda_{\mathrm{q}}$. In order to distinguish values measured at outer and inner target the notation $\lambda_{\mathrm{q}}^{\text {out }}$ and $\lambda_{\mathrm{q}}^{\text {in }}$ is used in the following. This is adapted when comparing to 

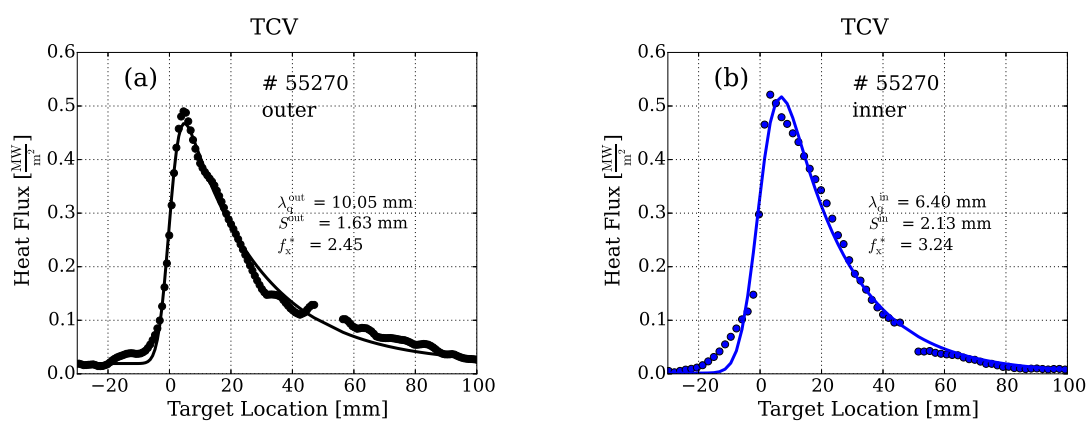

Figure 7. IR target profiles for \# $55270\left(\mathrm{He}\right.$, non-favourable, $\delta_{\mathrm{up}}=0$ ) together with a fit using (1) for (a) outer and (b) inner target.

previous results, typically using $\lambda_{\mathrm{q}}$ for the value measured at the outer target. The profiles for both targets are mapped to the outer mid-plane to have a common reference point. Single heat flux profiles are fitted for every $5 \mathrm{~ms}$ and the values averaged over typically $200 \mathrm{~ms}$ (40 profiles) for each discharge. Error bars are omitted in most figures for clarity. Asymmetries for $\lambda_{\mathrm{q}}$ have been reported in previous studies at e.g. ASDEX Upgrade [22, 16, 39], JET [26] and TCV [14] and are discussed separately.

Figure 8 shows the correlation between $\delta_{\mathrm{up}}$ and (a) $\lambda_{\mathrm{q}}^{\text {out }}$ and (b) $\lambda_{\mathrm{q}}^{\text {in }}$. The values
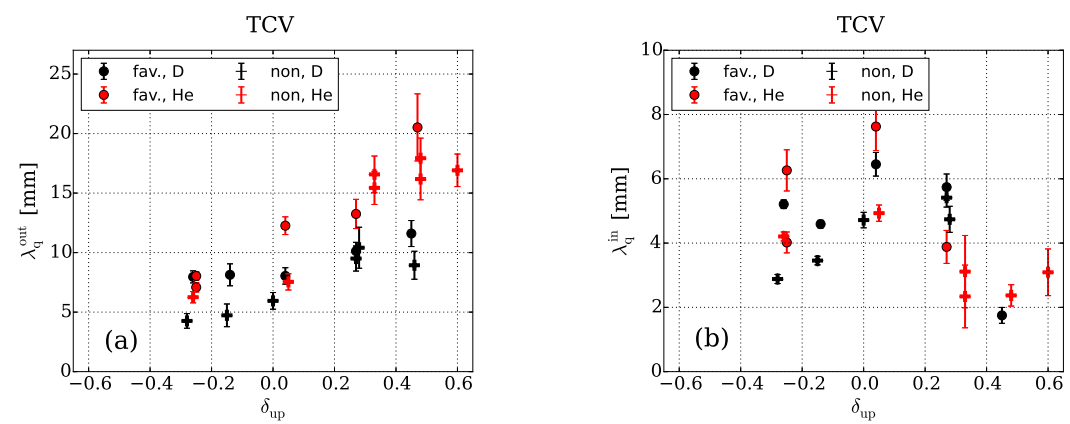

Figure 8. Correlation between $\delta_{\mathrm{up}}$ and (a) $\lambda_{\mathrm{q}}^{\text {out }}$ and (b) $\lambda_{\mathrm{q}}^{\text {in }}$.

for $\lambda_{\mathrm{q}}^{\text {out }}$ monotonically increase with increasing $\delta_{\mathrm{up}}$. The values for $\lambda_{\mathrm{q}}^{\text {in }}$ do not show a

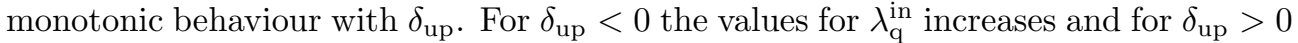
they decrease, having a maximum value around $\delta_{\text {up }} \approx 0$.

Figure 9 shows $\lambda_{\mathrm{q}}^{\text {in }}$ and $\lambda_{\mathrm{q}}^{\text {out }}$ depending on $\delta_{\mathrm{up}}$ for deuterium and favourable drift direction. They appear to diverge for $\delta_{\text {up }}>0$ and behave mostly indifferent for $\delta_{\text {up }}<0$.

\subsection{Further correlations of $\lambda_{\mathrm{q}}$}

\section{Magnetic drift direction}

The drift direction does not display notable influence on $\lambda_{\mathrm{q}}^{\text {out }}$ and $\lambda_{\mathrm{q}}^{\text {in }}$ for our database. 


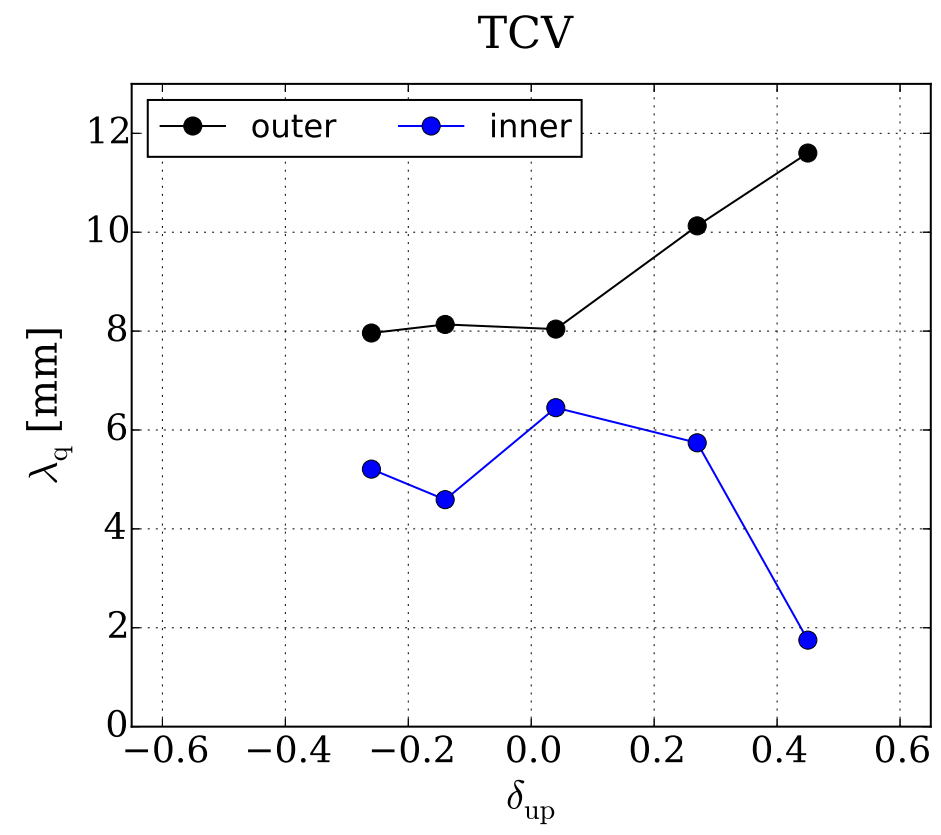

Figure 9. Power fall-off length $\lambda_{\mathrm{q}}$ dependent on the upper triangularity for both divertor targets in deuterium and favourable drift direction.

\section{Edge safety factor $q_{95}$}

Dedicated discharges with lower $\kappa$ are performed to vary $q_{95}$ independently of $\delta_{\text {up }}$, presented in table 2. This is aiming at a distinction between the effect of $\delta_{\text {up }}$ and $q_{95}$ on $\lambda_{\mathrm{q}}$. The change of $q_{95}$ at fixed $\delta_{\text {up }}$ does not affect $\lambda_{\mathrm{q}}^{\text {out }}$ outside of error bars. A change of $\delta_{\text {up }}$ at fixed $q_{95}$ on the contrary leads to a change of $\lambda_{\mathrm{q}}^{\text {out }}$. For $\lambda_{\mathrm{q}}^{\text {in }}$ no correlation outside the experimental uncertainty is found for this subset of discharges.

Since no TS data is available for discharge 52355 , no discrimination is possible between $q_{95}$ and $\delta_{\text {up }}$ for $T_{\text {e,edge }}$ effects in this data set.

Table 2. Discharge parameters for a selected subset of the database.

\begin{tabular}{c|r|c|c|c|c|c} 
Discharge number & \multicolumn{1}{c|}{$\delta_{\mathrm{up}}$} & \multicolumn{1}{c}{$q_{95}$} & $\kappa$ & \multicolumn{1}{c}{$\lambda_{\mathrm{q}}^{\text {out }}[\mathrm{mm}]$} & $\lambda_{\mathrm{q}}^{\text {in }}[\mathrm{mm}]$ & $T_{\mathrm{e}, \text { edge }}[\mathrm{eV}]$ \\
\hline 52307 & -0.15 & 2.49 & 1.34 & $4.7 \pm 1.0$ & $3.5 \pm 0.2$ & 107 \\
52315 & 0.27 & 3.29 & 1.53 & $9.5 \pm 1.0$ & $5.4 \pm 0.3$ & 64 \\
52355 & 0.28 & 2.46 & 1.29 & $10.4 \pm 1.7$ & $4.7 \pm 0.4$ & -
\end{tabular}

\section{Connection length}

Previous studies at TCV report an increase of $\lambda_{q}^{\text {out }}$ with increasing connection length $L_{\mathrm{OMP}}^{\text {out }}$ to the outer target by shifting the vertical position of the magnetic axis and hence increasing the distance between X-point and outer target $[13,14]$. Since $L_{\mathrm{OMP}}^{\text {out }}$ is fixed in our study, the variation of $\lambda_{\mathrm{q}}^{\text {out }}$ cannot be explained by a connection length variation.

The values for $\lambda_{\mathrm{q}}^{\text {in }}$ decrease for increasing $L_{\mathrm{OMP}}^{\mathrm{in}}$. Note, $L_{\mathrm{OMP}}^{\mathrm{in}}$ in the database is 
altered by varying $\delta_{\text {up }}$ which does not affect $L_{\mathrm{OMP}}^{\text {out }}$.

\section{Main ion species}

Helium shows on average larger values for $\lambda_{\mathrm{q}}^{\text {out }}$ compared to deuterium, in line with ASDEX Upgrade L-Mode [27] and JET H-Mode [28, 29] observations. For $\lambda_{\mathrm{q}}^{\text {in }}$ no difference is observed outside experimental uncertainties.

Edge electron temperature $T_{\mathrm{e}, \text { edge }}$

In figure 10 the dependence of $\lambda_{\mathrm{q}}^{\text {out }}$ and $\lambda_{\mathrm{q}}^{\text {in }}$ on $T_{\mathrm{e}, \text { edge }}$ is shown. Both, $\lambda_{\mathrm{q}}^{\text {out }}$ and $T_{\text {e,edge }}$, are changing when $\delta_{\mathrm{up}}$ is scanned. A larger $\lambda_{\mathrm{q}}^{\text {out }}$ is measured for lower $T_{\mathrm{e}, \text { edge }}$. At low $T_{\mathrm{e}, \text { edge }}$ a large variation in $\lambda_{\mathrm{q}}^{\text {in }}$ is observed, whereas at larger $T_{\mathrm{e} \text {,edge }}$ $\lambda_{\mathrm{q}}^{\text {in }}$ decreases for increasing $T_{\mathrm{e}, \text { edge }}$.
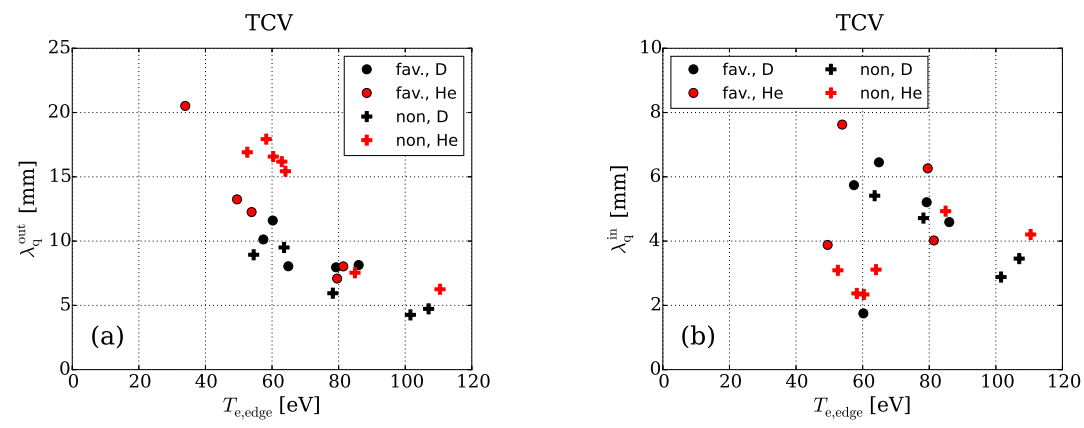

Figure 10. Correlation between $T_{\mathrm{e}, \text { edge }}$ and (a) $\lambda_{\mathrm{q}}^{\text {out }}$ and (b) $\lambda_{\mathrm{q}}^{\text {in }}$.

\section{Discussion}

\subsection{Comparison to empirical scaling laws}

Available scaling laws for $\lambda_{\mathrm{q}}^{\text {out }}$ in H-Mode based on a multi-machine database (not including TCV) show that the poloidal magnetic field at the outer mid-plane $B_{\text {pol }}^{\text {OMP }}$ is the main quantity determining $\lambda_{\mathrm{q}}^{\text {out }}[40]$.

$$
\lambda_{\mathrm{q}}^{\mathrm{Multi}}[\mathrm{mm}]=0.63 \cdot B_{\mathrm{pol}}^{\mathrm{OMP}}[\mathrm{T}]^{-1.19}
$$

The database discussed in this paper has a fixed plasma current and variations of $B_{\mathrm{pol}}$ due to the plasma shaping of less than $15 \%$. Although a multi-machine scaling for inner wall limited plasmas has been recently published [41], no multi-machine scaling for diverted L-Mode exists up to now. However, a scaling law for JET and ASDEX Upgrade was presented by Scarabosio et. al. [42]:

$$
\lambda_{\mathrm{q}}^{\text {Scarabosio }}[\mathrm{mm}]=1.44 \cdot B_{\text {tor }}[\mathrm{T}]^{-0.80} \cdot q_{95}^{1.14} \cdot P_{\mathrm{SOL}}[\mathrm{MW}]^{0.22} \cdot R[\mathrm{~m}]^{-0 .(33)}
$$

Since for JET and ASDEX Upgrade the L-Mode $\lambda_{\mathrm{q}}^{\text {out }}$ is about twice that of the HMode scaling $[42,16,39]$, we also compare the database to the scaling law presented by Eich et. al. [29] with an additional pre factor of 2 .

$$
\lambda_{\mathrm{q}}=2 \cdot \lambda_{\mathrm{q}}^{\mathrm{Eich}}[\mathrm{mm}]=2 \cdot 0.73 \cdot B_{\mathrm{tor}}[\mathrm{T}]^{-0.78} \cdot q_{\mathrm{cyl}}^{1.2} \cdot P_{\mathrm{SOL}}[\mathrm{MW}]^{0.1} \cdot R[\mathrm{~m}]^{0 \cdot 99}
$$

The measured values versus these scaling law predictions are shown in figure 11. All three scaling laws, using global discharge parameters, disagree in absolute magnitude 
as well as in the increase of $\lambda_{\mathrm{q}}^{\text {out }}$ with increasing $\delta_{\mathrm{up}}$. Using an additional pre factor of 2 for the multi-machine H-Mode scaling (2) as well, shown in figure 11(a) with a blue line, matches about the average value for deuterium. Note, that the databases used for this scaling laws do not contain dedicated triangularity scans.

A more recent study at ASDEX Upgrade by Sieglin et. al. [39] revealed that $\lambda_{\mathrm{q}}^{\text {out }}$ in
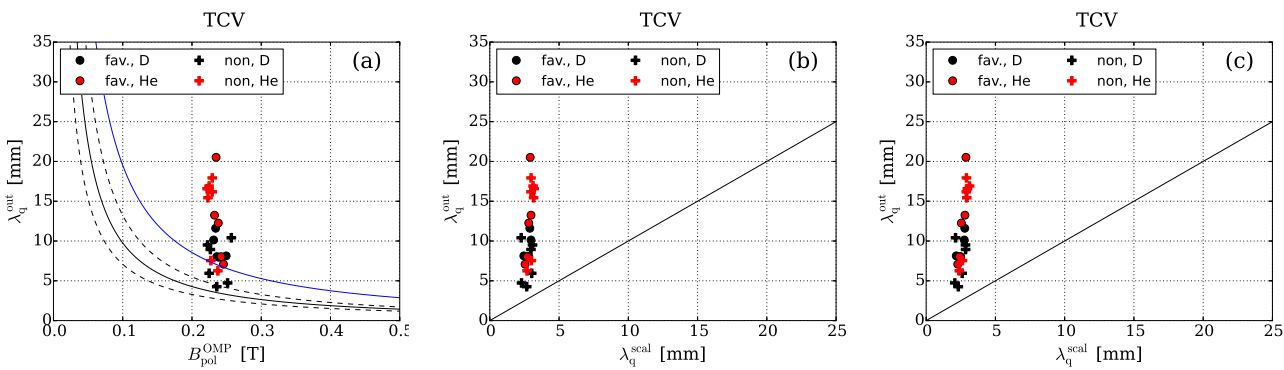

Figure 11. Comparison between measured $\lambda_{\mathrm{q}}^{\text {out }}$ and empirical scaling laws. (a) multi-machine H-Modescaling (2) (black) with typical database scatter of $25 \%$ (dashed black) and $2 \times$ scaling prediction (blue), (b) $2 \times$ Eich H-Mode scaling (4) and (c) Scarabosio L-Mode scaling (3).

L-Mode is dependent not only on $B_{\text {pol }}$ but also on stored energy $W_{\text {MHD }}$, edge electron density $\bar{n}_{\mathrm{e}, \text { edge }}$ and main ion mass $A$ (in atomic units u):

$\lambda_{\mathrm{q}}^{\text {Sieglin }}[\mathrm{mm}]=0.16 \cdot B_{\mathrm{pol}}[\mathrm{T}]^{-0.66} \cdot A[\mathrm{u}]^{-0.15} \cdot\left(\frac{W_{\mathrm{MHD}}[\mathrm{MJ}]}{\bar{n}_{\mathrm{e}, \text { edge }}\left[10^{19} \mathrm{~m}^{-3}\right]}\right)^{-0.93}$

with $B_{\text {pol }}=\frac{\mu_{0} I_{\mathrm{p}}}{2 \pi a \sqrt{\frac{1+\kappa^{2}}{2}}}$ and minor radius $a$. The dependence on $W_{\mathrm{MHD}} / \bar{n}_{\mathrm{e}, \text { edge }}$ is interpreted to be a dependence on $T_{\text {e,edge }}[39]$. The edge density is measured with the edge channel of the interferometer in this ASDEX Upgrade study. A linear correlation between $W_{\mathrm{MHD}} / n_{\mathrm{e}, \text { edge }} / V$ and $T_{\mathrm{e} \text {,edge }}$ is shown in figure 12 for the presented TCV database with $V$ being plasma volume.

The plasma volume is not changing significantly for the presented database for TCV but is used to keep the same physical dimension and account for the size dependence of the stored energy. We generalize the ASDEX Upgrade scaling by using $T_{\text {e,edge }}$ instead of $W_{\mathrm{MHD}} / \bar{n}_{\mathrm{e} \text {,edge }}$. The pre factor is adapted using a linear regression between $T_{\mathrm{e} \text {,edge }}$ at $\rho_{\text {pol }}=0.95$ and $W_{\mathrm{MHD}} / \bar{n}_{\mathrm{e} \text {,edge }}$ for the ASDEX Upgrade database.

$\lambda_{\mathrm{q}}[\mathrm{mm}]=163 \cdot B_{\mathrm{pol}}[\mathrm{T}]^{-0.66} \cdot A[\mathrm{u}]^{-0.15} \cdot T_{\mathrm{e}, \mathrm{edge}}[\mathrm{eV}]^{-0.93} \cdot\left(\frac{R}{R_{\mathrm{AUG}}}\right)^{x}$

The unknown major radius $R$ dependence $\left(R^{x}\right)$ due to the single machine scaling law is explicitly taken into account. The exponent of the major radius $R$ is found to be -0.03 in order to match the absolute values for deuterium with favourable drift direction in our TCV database using the same exponents otherwise, see figure 13.

$\lambda_{\mathrm{q}}^{\mathrm{L}-\text { Mode }}[\mathrm{mm}]=165 \cdot B_{\mathrm{pol}}[\mathrm{T}]^{-0.66} \cdot A[\mathrm{u}]^{-0.15} \cdot T_{\mathrm{e}, \text { edge }}[\mathrm{eV}]^{-0.93} \cdot R[\mathrm{~m}]^{-0.03}$

The pre factor contains the value of $R_{\mathrm{AUG}}^{0.03}$. Deuterium discharges with non-favourable drift direction are about $22 \%$ lower than the scaling prediction. Helium discharges with favourable (non-favourable) drift direction are about 15\% (55\%) above the scaling predictions. 


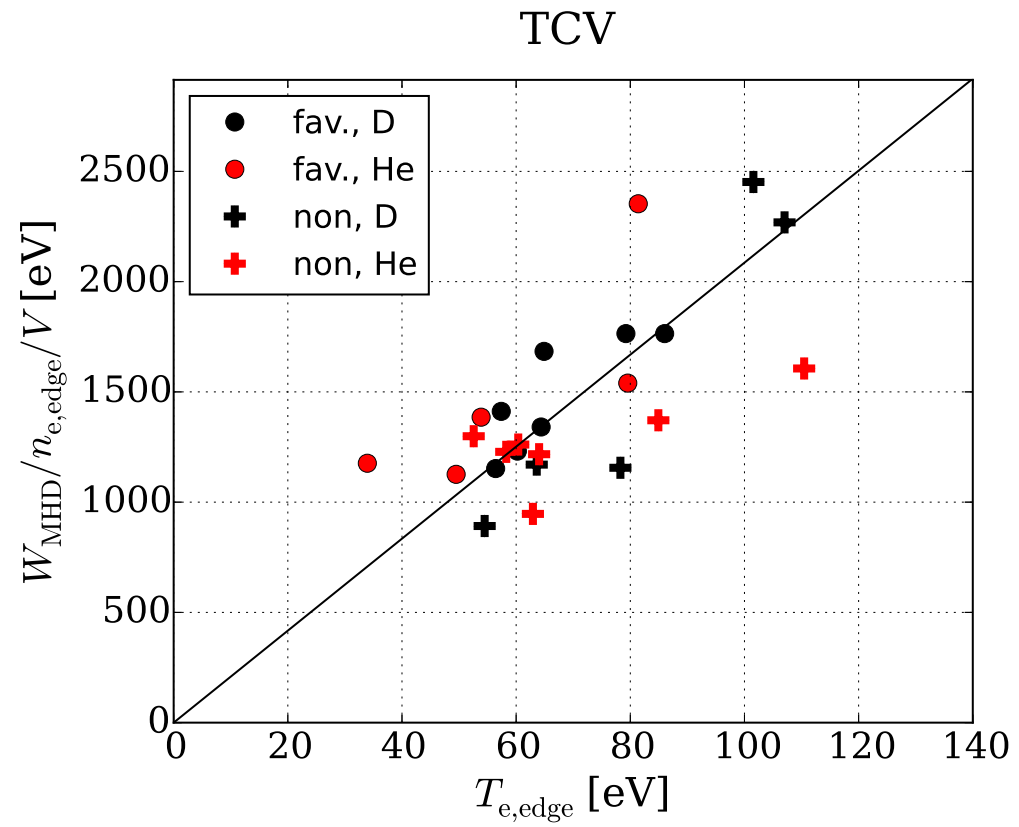

Figure 12. Correlation between edge electron temperature $T_{\mathrm{e}, \text { edge }}$ and stored energy divided by edge electron density and plasma volume $W_{\mathrm{MHD}} / n_{\mathrm{e}, \text { edge }} / V$.

A scaling based uniquely on global machine parameters, e.g. $B_{\text {pol }}$, is not able to reproduce the variation of $\lambda_{\mathrm{q}}^{\text {out }}$ with the measured factor of four in this L-Mode TCV database. The $R^{-0.03}$ dependence is in line with multi-machine regression results for H-Mode as well as the L-Mode scaling for JET and ASDEX Upgrade. However, one has to be careful when extrapolating from (7) to different devices because the parameter range, especially for $B_{\mathrm{pol}}$, in the ASDEX Upgrade data base is limited, see [37]. In order to improve this scaling, re-analysis by collecting multi-machine data is needed. Especially data from JET is essential to be able to extrapolate towards larger machines like ITER. However, this multi-machine analysis is outside of the scope of this paper, which provides a first hint towards the underlying mechanism for the L-Mode power fall-off length $\lambda_{\mathrm{q}}$.

\subsection{Turbulent transport}

The enhanced energy confinement with negative triangularity has been reported to be caused by reduced turbulent transport in the confined region [17, 18, 21]. This is in line with the increased $T_{\mathrm{e} \text {,edge }}$ and stored energy $W_{\mathrm{MHD}}$ at constant heating power in the presented TCV database.

Recently, reduced turbulent transport has been reported for the scrape-off layer in elongated limited plasmas with negative triangularity using linear and nonlinear simulations [31]. This is in qualitative agreement with the observed decreasing $\lambda_{\mathrm{q}}^{\text {out }}$ with decreasing $\delta_{\text {up }}$. In the presented database only $\delta_{\text {up }}$ was changed and the lower part of the plasma was kept constant with a positive $\delta_{\text {low }}$. A more detailed comparisons with these turbulence simulations are proposed to investigate the influence of $\delta_{\text {up }}$ on 


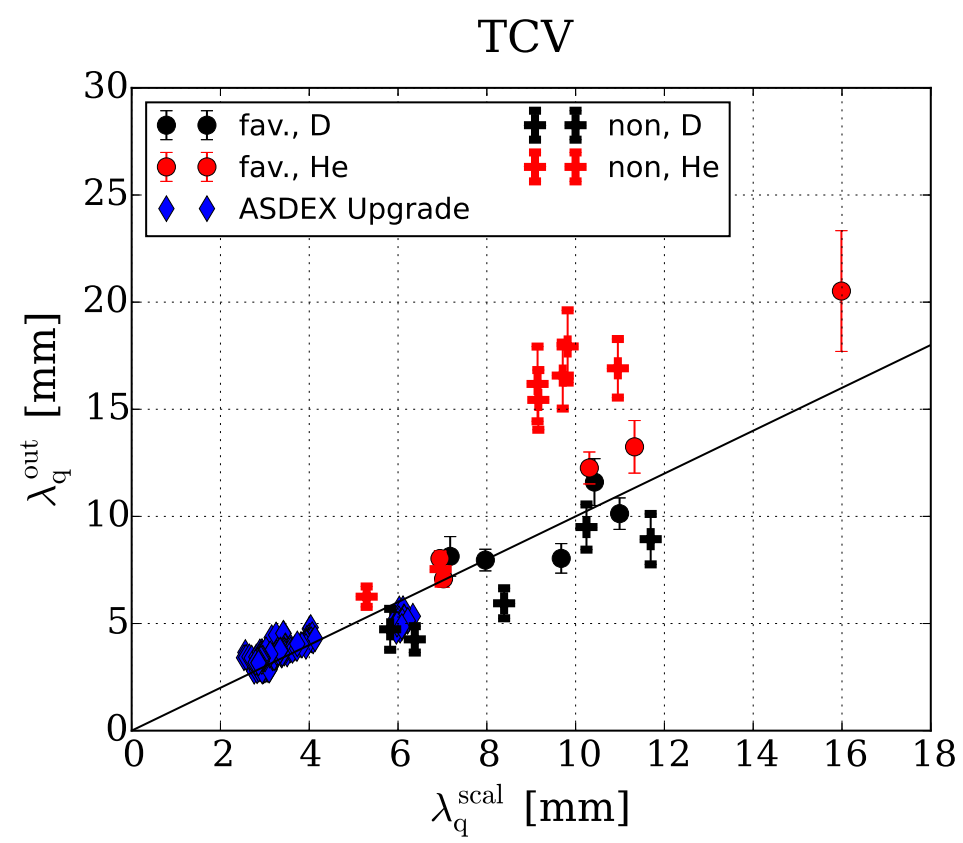

Figure 13. $\lambda_{\mathrm{q}}^{\text {out }}$ versus L-Mode scaling law (7). The ASDEX Upgrade data is taken from [39].

turbulence and its link to $\lambda_{\mathrm{q}}$ in diverted plasma configurations.

The experimental data does not allow to distinguish if the triangularity directly effects $\lambda_{\mathrm{q}}$ or indirectly by changing $T_{\mathrm{e} \text {,edge }}$ due to the correlation between $\delta_{\mathrm{up}}$ and $T_{\mathrm{e} \text {,edge }}$.

\subsection{Negative triangularity reactor}

A negative triangularity reactor concept has been discussed in recent years, e.g. [5, 6 , 7]. One of the key advantages of such a device is the possibility of the divertor region being at a larger major radius $R_{\text {div }}$ and with this having a larger toroidal divertor extent $\left(2 \pi R_{\text {div }} f_{\text {tor }}\right.$, with $f_{\text {tor }}$ toroidal wetted fraction) for heat exhaust. The wetted area $\mathrm{A}$ is:

$$
A=2 \pi R_{\text {div }} f_{\text {tor }} \lambda_{\text {int }, \mathrm{t}}
$$

with $\lambda_{\text {int, } t}$ being the width of the profile at the divertor target. For an attached, conventional divertor using the heat flux characterization presented in (1), the target fall-off length is given by [43]

$$
\lambda_{\text {int }, \mathrm{t}}=f_{\mathrm{x}}^{*}\left(\lambda_{\mathrm{q}}+1.64 S\right)
$$

In the presented study the lower triangularity was kept constant in order to keep the divertor geometry constant, thus, $R_{\text {div }}$ was unchanged. However, $\lambda_{\mathrm{q}}^{\text {out }}$ decreased for decreasing $\delta_{\text {up }}$. This potentially counteracts the beneficial effect of negative triangularity leading to a larger $R_{\text {div }}$ with decreasing $\delta_{\text {low }}$. Additionally, a narrower scrape-off layer decreases the capability to radiate power by impurity seeding in the scrape-off layer. Our experiments, however, may not be fully representative for a plasma shape with both, negative upper and lower triangularity. Future studies with 
similar experiments but changing also the lower triangularity are needed in order to investigate the effect of both triangularities and the increased toroidal divertor extent.

\subsection{Comparison to heuristic drift-based model}

A prominent edge heat transport model is the heuristic drift-based model by Goldston [30]. This model predicts, independent of the actual value for the parallel flow velocity, a ratio of inner and outer power fall-off length $\lambda_{\mathrm{q}}$ of (equation (21) from ref. [16]):

$$
\frac{\lambda_{\mathrm{q}}^{\text {in }}}{\lambda_{\mathrm{q}}^{\text {out }}}=\frac{1-\delta_{\mathrm{x}}}{1+\delta_{\mathrm{x}}}
$$

were ' $x$ ' denotes upper or lower triangularity, dependent on the vertical drift direction of electrons or ions. Here it is assumed that $T_{\mathrm{e}}$ at the outer and inner mid-plane separatrix position are equal as well as that the averaged parallel flow velocity is the same. Figure 14 shows the comparison between (10) and the ratio of the power fall-off lengths, using the upper triangularity for $\delta_{\mathrm{x}}$. For positive $\delta_{\mathrm{up}}$ the ratio increases for

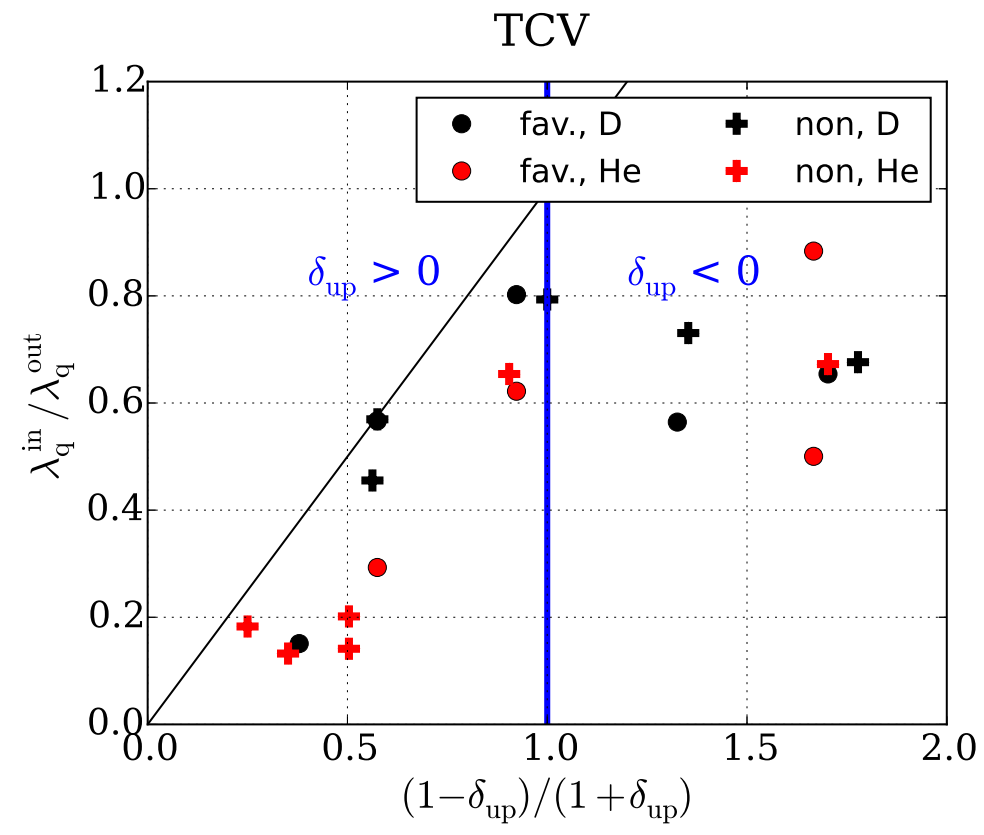

Figure 14. $\lambda_{\mathrm{q}}$ ratio depending on the triangularity factor (10) using the upper triangularity.

decreasing $\delta_{\text {up }}$, saturating at a value around 0.7 for negative $\delta_{\text {up }}$. The data for $\delta_{\text {up }}>0$ shows agreement to the prediction using $\delta_{\text {up }}$. A drift-based model, as discussed here, predicts changes with the vertical drift direction. Using for both field direction either ion or electron drift direction in (10) results in diverse defining triangularity. This is in contrast to the measurement, the ratio between inner and outer $\lambda_{\mathrm{q}}$ shows no clear difference between the two field directions. For both field directions the ratio changes with the upper triangularity. 
The saturation in the ratio for $\delta_{\mathrm{up}}<0$ in the experiment is in disagreement with the heuristic drift-based model prediction. From the model a linear decrease of $\lambda_{q}^{\text {out }}$ is predicted for decreasing $\delta$, in line with the measurement. A linear increase of $\lambda_{\mathrm{q}}^{\text {in }}$ is predicted for decreasing $\delta$, only observed for $\delta_{\text {up }}>0$. For $\delta_{\text {up }}<0$ the model predicts $\lambda_{\mathrm{q}}^{\text {in }}>\lambda_{\mathrm{q}}^{\text {out }}$, not observed in this experiment.

\section{Conclusions}

Shaping the plasma boundary can change the power fall-off length beyond established empirical multi-machine scaling laws typically using global plasma parameters without dedicated shaping scans. Extending an L-Mode scaling law from ASDEX Upgrade [39] reveals a dependence on edge electron temperature $T_{\mathrm{e} \text {,edge }}$ in both machines and no explicit size dependence. Changing the upper triangularity leads to changes in confinement as well as the power fall-off length in the presented TCV L-Mode database. Enhanced energy confinement at negative triangularity, explained by a reduction of turbulent transport in the confined region [21], is confirmed. The effect of triangularity on scrape-off layer turbulence could be a possible explanation for smaller $\lambda_{\mathrm{q}}^{\text {out }}$ for decreasing $\delta_{\mathrm{up}}$, in qualitative agreement with turbulence simulation in limited plasmas [31].

Helium discharges exhibit a larger $\lambda_{\mathrm{q}}$ compared to deuterium, in line with previous studies on JET $[28,29]$ and ASDEX Upgrade [27]. Reversal of the vertical drift direction has no significant influence on both, $\lambda_{\mathrm{q}}^{\text {out }}$ and $\lambda_{\mathrm{q}}^{\text {in }}$, in the presented database. The power fall-off length measured at the inner divertor target exhibits a non monotonic behaviour for changing $\delta_{\mathrm{up}}$. For $\delta_{\mathrm{up}} \approx 0$ the largest $\lambda_{\mathrm{q}}^{\text {in }}$ are obtained. The asymmetry between inner and outer $\lambda_{\mathrm{q}}$ is compared to an interpretation [16] of the heuristic drift-based model [30]. Especially for negative $\delta_{\text {up }}$ no agreement is found. Considerations of a negative triangularity reactor due to reduced core turbulence and larger major radius of the divertor $R_{\mathrm{div}}$ are using $\lambda_{\mathrm{q}}$ from scaling laws deduced from positive triangularity discharges. The effect of a potentially smaller $\lambda_{\mathrm{q}}$ for negative $\delta$ needs to be taken into account for an overall assessment of such novel configurations. Further studies are needed to assess the effect of changing the connected, lower triangularity in order to increase $R_{\text {div }}$.

\section{Acknowledgements}

This work has been carried out within the framework of the EUROfusion Consortium and has received funding from the Euratom research and training programme 20142018 under grant agreement No 633053. The views and opinions expressed herein do not necessarily reflect those of the European Commission.

\section{References}

[1] LOARTE, A. et al., Nuclear Fusion 47 (2007) S203.

[2] PITTS, R. et al., Journal of Nuclear Materials 438 (2013) S48.

[3] KUKUSHKIN, A. et al., Journal of Nuclear Materials 438 (2013) S203.

[4] GOLdSTON, R. et al., Plasma Physics and Controlled Fusion 59 (2017) 055015.

[5] KIKUCHI, M. et al., Negative triangularity as a possible tokamak scenario, in JPS Conf. Proc., volume 1, page 015014, 2014.

[6] MEDVEDEV, S. Y. et al., Nuclear Fusion 55 (2015) 063013.

[7] SAUTER, O., Fusion Engineering and Design (2016). 
[8] HOFMAnN, F. et al., Plasma Physics and Controlled Fusion 36 (1994) B277.

[9] CODA, S. et al., Nuclear Fusion 57 (2017) 102011.

[10] PIRAS, F. et al., Plasma Physics and Controlled Fusion 51 (2009) 055009.

[11] REIMERDES, H. et al., Plasma Physics and Controlled Fusion 55 (2013) 124027.

[12] THEILER, C. et al., Nuclear Fusion 57 (2017) 072008.

[13] GALLO, A. et al., Nuclear Materials and Energy (2016).

[14] MAURIZIO, R. et al., Nuclear Fusion accepted (2017).

[15] GAllO, A. et al., Plasma Physics and Controlled Fusion accepted (2017).

[16] FAITSCH, M. et al., Plasma Physics and Controlled Fusion 57 (2015) 075005.

[17] POCHELON, A. et al., Plasma and Fusion Research 7 (2012) 2502148.

[18] CAMENEN, Y. et al., Nuclear Fusion 47 (2007) 510.

[19] POCHELON, A. et al., Nuclear Fusion 39 (1999) 1807.

[20] MORET, J.-M. et al., Physical Review Letters 79 (1997) 2057.

[21] MARINONI, A. et al., Plasma Physics and Controlled Fusion 51 (2009) 055016.

[22] EICH, T. et al., Plasma Physics and Controlled Fusion 47 (2005) 815.

[23] AHO-MANTILA, L. et al., Nuclear Fusion 52 (2012) 103006.

[24] LIU, S. et al., Physics of Plasmas 19 (2012) 042505.

[25] ZHANG, B. et al., Fusion Engineering and Design 105 (2016) 70.

[26] PITTS, R. A. et al., Journal of Nuclear Materials 337339 (2005) 146.

[27] SIEGLIN, B. et al., Nuclear Materials and Energy (2016).

[28] FUNDAMENSKI, W. et al., Nuclear fusion 51 (2011) 083028.

[29] EICH, T. et al., Physical Review Letters 107 (2011) 215001.

[30] GOLDSTON, R. J., Nuclear Fusion 52 (2012) 013009.

[31] RIVA, F. et al., Plasma Physics and Controlled Fusion 59 (2017) 035001.

[32] HOFMANN, F. and TONETTI, G., Nuclear Fusion 28 (1988) 1871.

[33] SHEIKH, U. et al., Review of Scientific Instruments 87 (2016) 11D431.

[34] FRANKE, S., Application of Thomson scattering at 1.06mm as a diagnostic for spatial profile measurements of electron temperature and density on the TCV tokamak, PhD thesis, EPFLLausanne, Switzerlandy, 1997.

[35] SAUTER, O. et al., Physics of Plasmas (1994-present) 21 (2014) 055906.

[36] HERRMANN, A. et al., Plasma Physics and Controlled Fusion 37 (1995) 17.

[37] SIEGLIN, B. et al., Review of Scientific Instruments 86 (2015).

[38] PITTS, R. et al., Nuclear Fusion 43 (2003) 1145.

[39] SIEGLIN, B. et al., Plasma Physics and Controlled Fusion 58 (2016) 055015.

[40] EICH, T. et al., Nuclear Fusion 53 (2013) 093031.

[41] HORACEK, J. et al., Plasma Physics and Controlled Fusion 58 (2016) 074005.

[42] SCARABOSIO, A. et al., Journal of Nuclear Materials 438, Supplement (2013) S426 .

[43] MAKOWSKI, M. A. et al., Physics of Plasmas (1994-present) 19 (2012) 056122. 